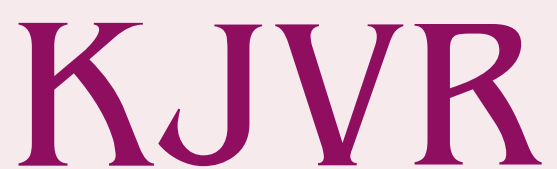

Korean Journal of Veterinary Research

\section{Case Report}

pISSN 2466-1384 · elSSN 2466-1392

Korean J Vet Res 2021;61(3):e28

https://doi.org/10.14405/kjvr.2021.61.e28

*Corresponding author:

Byeong-Teck Kang

Laboratory of Veterinary Internal Medicine,

College of Veterinary Medicine, Chungbuk

National University, 1 Chungdae-ro,

Seowon-gu, Cheongju 28644, Korea

Tel: +82-43-261-3744

Fax: +82-43-267-2595

E-mail: kangbt@chungbuk.ac.kr

ORCID:

https://orcid.org/0000-0002-4471-4342

Conflict of interest:

The authors declare no conflict of interest.

Received: July 1, 2021

Revised: August 19, 2021

Accepted: August 23, 2021

\title{
Cutaneous angiomatosis in a dog: a case report
}

\author{
Yoonhoi Koo, Taesik Yun, Yeon Chae, Dohee Lee, Hakhyun Kim, \\ Mhan-Pyo Yang, Byeong-Teck Kang
}

Laboratory of Veterinary Internal Medicine, College of Veterinary Medicine, Chungbuk National University, Cheongju 28644, Korea

A 1-year-old castrated mixed-breed dog presented with diffuse, purple lesions arranged in an irregular patchy pattern, with a slight elevation on the right hindlimb extending from the tarsus joint to the upper region of the thigh. Dermatological examinations and fungal and bacterial cultures revealed no infectious agents. The therapeutic response to antibiotics and antifungal agents was negative. A histopathology examination of the lesion revealed vascular proliferation with vasodilation and numerous varying-sized vessels. Mast-cell-dominated perivascular cuffing was also noted. The dog was diagnosed with cutaneous angiomatosis due to diffuse lesions and the histopathology findings of hemangioma.

Keywords: dogs; angiomatosis; hemangioma; neoplasms; vascular malformations; case report

Angiomatosis and vascular tumors, including hemangioma, hemangiosarcoma, lymphangiosarcoma, and vascular hamartoma, are characterized by endothelial cell proliferation [1]. Angiomatosis is a rare benign vascular proliferative change that can be induced by bacterial infections, the repair response to a primary injury to damaged tissue, or an unknown origin [2-4]. Angiomatosis lesions have been reported to arise in the skin, viscera, vertebrae, meninges, intestine, and ovary [3]. Cutaneous angiomatosis is a rare disease in veterinary medicine, with only seven dogs with cutaneous angiomatosis reported [2,5-9]. Surgical excision methods, such as conventional surgery, coagulation laser therapy, and radiation, are the only treatments for cutaneous angiomatosis [6-8].

To the best of the authors' knowledge, canine cutaneous angiomatosis has never been reported in Korea. This case report describes canine cutaneous angiomatosis diagnosed based on the clinical features, dermatological findings, and histopathology findings.

A 1-year-old castrated male mixed-breed dog presented to Chungbuk National University Veterinary Teaching Hospital for gradually enlarging purple lesions on the right hindlimb that had developed 9 months prior (Fig. 1). The lesions extended from the tarsus joint to the thigh and were elevated, with an irregular patchy pattern of discoloration. The dog had no history of trauma involving the right hindlimb, and whole-body radiography revealed no fractures or other abnormalities induced by trauma. Furthermore, the lesions did not induce pain or lameness. The results of the skin cytological evaluation for infectious microorganisms and superficial and deep skin scrapings were negative. A complete blood count revealed no remarkable findings.

Hyperproteinemia (7.5 g/dL; reference interval, 5.4-7.1 g/dL) and hyperalbu- 

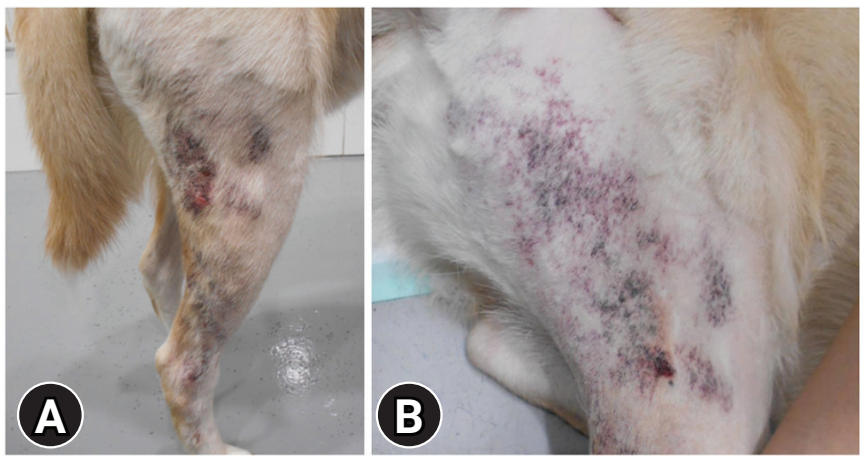

Fig. 1. Right hindlimb of a dog with cutaneous angiomatosis. (A) Multiple, diffuse, red to purple, discolored lesions were observed generally on the right hindlimb. (B) The lesions were observed as an irregular patchy pattern with slight elevation.

minemia (3.5 g/dL; reference interval, 2.6-3.3 g/dL) were observed on serum biochemical analysis; on the other hand, C-reactive protein and creatine phosphokinase levels were not increased. Despite the negative skin cytology results for microorganisms, deep pyoderma and dermatophytosis were not completely excluded because of the widespread lesions on the skin, including the dermis.

Bacterial and fungal cultures were performed for diagnosis because of the possibility of a false negative skin cytology result due to an incomplete skin test. Itraconazole (Ashicona; ALS, India) $10 \mathrm{mg} / \mathrm{kg}$ once daily and amoxicillin/clavulanate (Lactamox; A Progen Pharma, Korea) $25 \mathrm{mg} / \mathrm{kg}$ twice daily were prescribed for a therapeutic diagnosis of deep pyoderma and dermatophytosis until the culture was completed. On the other hand, the skin lesions on the right hindlimb did not improve, and the bacterial and fungal cultures were negative. Therefore, infectious skin diseases were excluded, and antibiotics and antifungal agents were discontinued. Histopathology analysis was performed on punch biopsy specimens obtained from the right hindlimb lesions. After collection, the samples were fixed in a $10 \%$ phosphate-buffered formalin solution. Hematoxylin and eosin (H\&E) staining was performed before optical microscopy, which revealed the proliferation of vessels with vasodilation and inflammation (Fig. 2). Diffuse vascular proliferation consisted of numerous capillaries of variable diameters in the dermis, irregular filling of blood cells and fibrin within the proliferated blood vessels, and perivascular cuffing in the upper region of the dermis. In the perivascular cuffing region, mast cells were observed mainly among the inflammatory cells, and the infiltration of plasma cells was also observed (Fig. 3). Microorganisms, such as bacteria and parasites, were not identified. Pleomorphism was not sufficient to indicate malignancy. The histo-
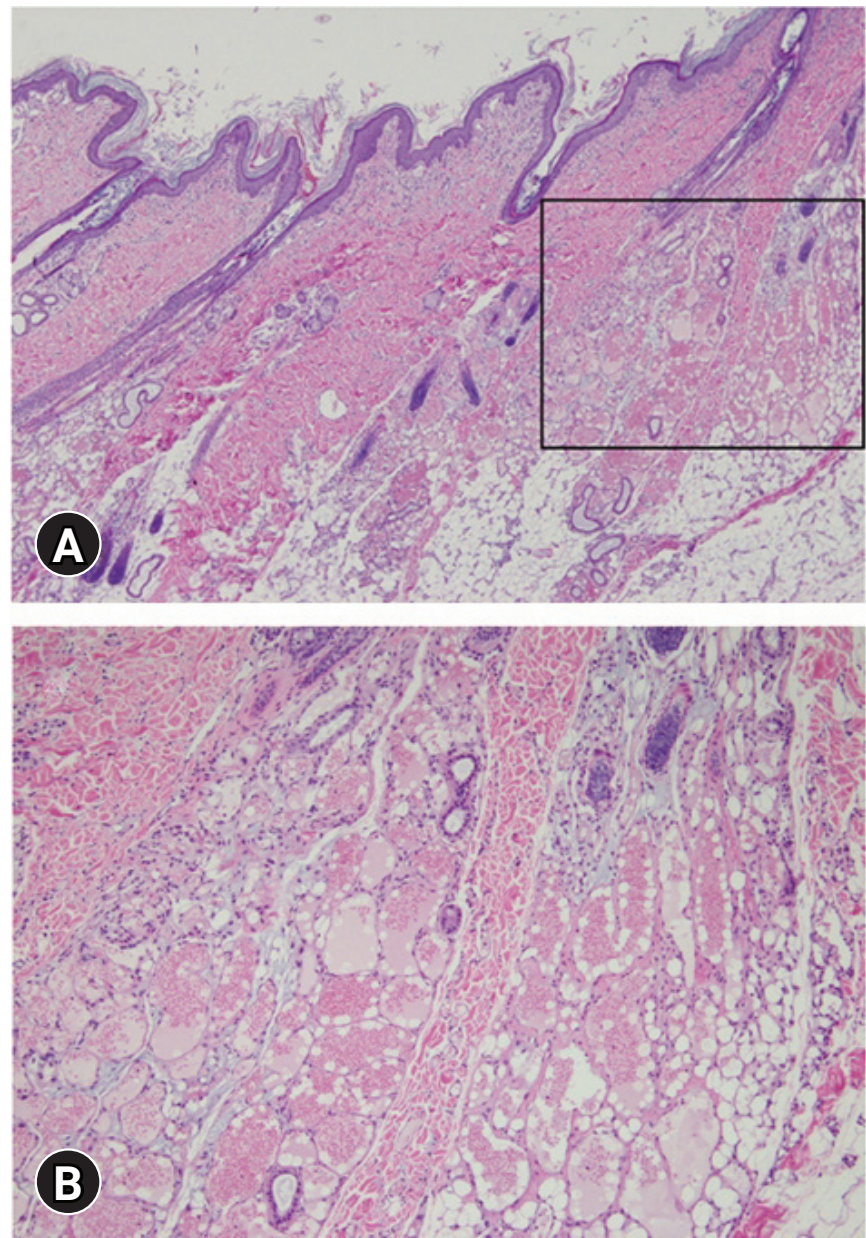

Fig. 2. Optical microscopy findings of the right hindlimb lesion. (A) Vascular proliferation and vasodilation in the upper dermis $\left(H \& E_{1}\right.$ $\times 40$ ); inset square represents (B). (B) The hemangioma pattern was observed in the lower part of the dermis, where vascular proliferation and vasodilation were connected in the hair follicle $\left(H \& E_{1}\right.$ $\times 100)$.

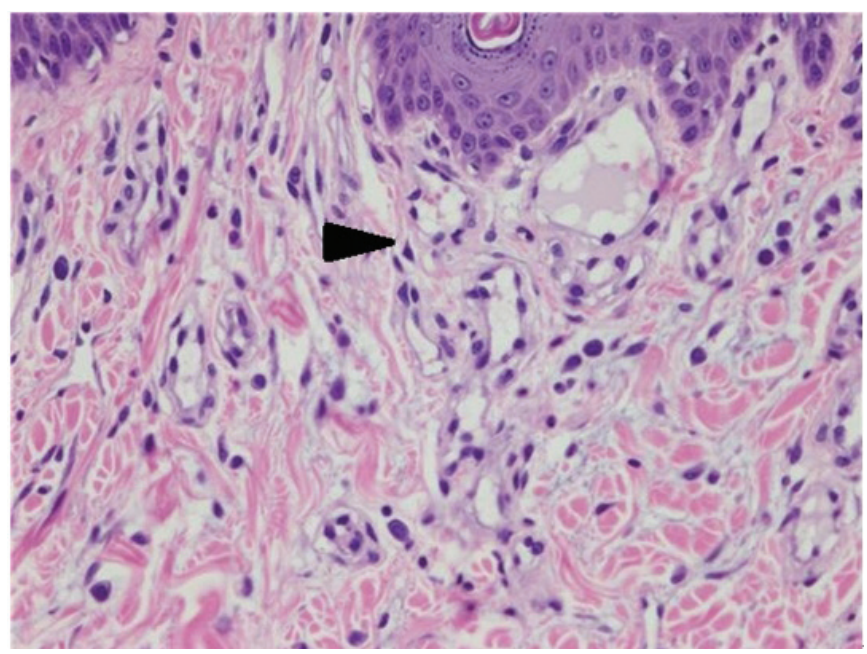

Fig. 3. Microscopy findings of the right hindlimb lesion. Inflammatory cells were mainly mast cells (arrowhead) $\left(H \& E_{1} \times 400\right)$. 
logical findings suggested hemangioma. On the other hand, the dog was younger than expected for hemangioma, and the lesions appeared diffuse and inconsistent with hemangiomas. Vasculitis was not ruled out because of perivascular inflammation, and $0.5 \mathrm{mg} / \mathrm{kg}$ of prednisolone (Solondo; Yuhan, Korea) twice daily for 2 weeks was prescribed. On the other hand, no clinical improvement was observed during the follow-up. Therefore, the dog was diagnosed with angiomatosis considering the presence of diffuse lesions, histology findings of blood vessel proliferation, and the absence of a response to treatment for vasculitis and infection. After that, the dog did not present to the veterinary hospital and could not receive additional treatment, including laser phototherapy. The total follow-up period was 45 days.

Cutaneous angiomatosis is typically characterized by variable-sized blood-filled vascular structures separated by apparently normal or myxomatous mesenchymal tissue in the dermal and subcutaneous regions [10]. Fibroblasts, pericytes, and smooth muscle cells, accompanied by proliferating endothelial cells, can be seen in some forms of angiomatosis [1]. Diseases with the characteristics of vascular proliferation, such as hamartoma, vascular hyperplasia, hemangioma, and hemangiosarco$\mathrm{ma}$, should be ruled out for a diagnosis of angiomatosis [7].

The right hindlimb lesions in this dog were elevated with diffuse red to purple discoloration, similar to the gross lesion reported previously in canine cutaneous angiomatosis [2]. Cutaneous angiomatosis in humans usually occurs in the lower extremities, followed by the chest wall, abdomen, and upper extremities [11]. On the other hand, the reported dog also had lesions on the extremities [2]. This case also coincides with previous reports in that the lesions occurred in the right hindlimb.

The histology findings of the previously reported dog with cutaneous angiomatosis included multiple capillary vessels and endothelial-lined cavernous vessels scattered and infiltrated throughout the superficial and deep dermis, surrounding adnexa, and extending to the subcutaneous adipose tissue and muscle [2]. Two major histological patterns of angiomatosis can be found in humans. The first is the haphazard proliferation of varying-sized vessels and clusters of capillary vessels adjacent to the vein walls. The second is a central large vessel surrounded by clusters of capillary-sized vessels arranged in nodules [11]. In the present case, mild spongiosis was observed in the epithelium, and vascular proliferation with vasodilation of the upper dermis and inflammatory cell infiltration into the peripheral tissues were observed. Most of the infiltrated inflammatory cells were identified as mast cells, but plasma cells were also identified. Vascular proliferation with vasodilation as a histologic pat- tern of hemangioma was observed in the lower part of the hair follicle of the dermis. The cells lining the vessels were identified as well-differentiated endothelial cells. These histopathological findings were consistent with previous findings in humans and dogs with cutaneous angiomatosis $[2,11]$.

The present case showed mast cell-based perivascular inflammatory cell infiltration, which was not a previously reported histology finding of angiomatosis. Several mast cell-derived mediators, including vascular endothelial growth factor, basic fibroblast growth factor, transforming growth factor- $\beta$, tumor necrosis factor- $\alpha$, and interleukin- 8 , are angiogenic and regulate endothelial cell proliferation [12]. Furthermore, mast cells may play important roles in the development and progression of canine cutaneous hemangioma, hemangiosarcoma, mammary adenoma, and adenocarcinoma [13]. In this case, the infiltration of mast cells around the blood vessels is thought to be due to excessive vascular proliferation. On the other hand, because immune-mediated diseases, including vasculitis, could not be ruled out, an immunomodulatory dose of prednisolone was prescribed for a therapeutic diagnosis, but the lesions did not improve. Therefore, immune-mediated diseases were excluded from the differential diagnosis.

Cutaneous hemangioma has a high incidence in older dogs. The appearance of a solitary, round, well-circumscribed, firm to fluctuant, raised bluish-to-reddish black dermal or subcutaneous growth ranging from 0.5 to $4 \mathrm{~cm}$ in diameter has been reported [14]. In this case, vascular proliferation was identified through histopathology. On the other hand, malignancy was not observed, suggesting the possibility of diffuse hemangioma, indicating cutaneous angiomatosis $[2,9,10]$. Hemangiosarcoma was also excluded from the differential diagnosis, considering the appearance of the lesion and the absence of findings of malignancy on histopathology.

Although bacterial infection and trauma can cause angiomatosis, angiomatosis without specific causes has also been reported [2-4]. Bacillary angiomatosis is one of the most common types of angiomatosis caused by bacterial infections [4]. Therefore, bacillary angiomatosis was a differential diagnosis in this dog. On the other hand, bacillary angiomatosis did not correspond to this case because no infectious agents were found in the dermatologic examination or histopathology. Trauma-induced angiomatosis was also excluded based on the patient's history, physical examination, and radiology investigation.

The results of the blood analysis were unremarkable. Hyperproteinemia with hyperalbuminemia in this dog was suspected of having resulted from dehydration. Therefore, this case was presumed to be a cutaneous angiomatosis of unknown origin. 
The dog was finally diagnosed with cutaneous angiomatosis of an unknown origin based on the signalment, gross lesions, exclusion of other diseases, and histopathology results. A previously reported dog with progressive cutaneous angiomatosis presented with slow progression over approximately 10 years [7]. Because the follow-up period was also short (45 days), it was impossible to accurately determine if the cutaneous angiomatosis in this dog was progressive.

This case report has several limitations. First, the cause of cutaneous angiomatosis was not identified. A Bartonella infection was not excluded because tests to identify Bartonella, such as the Warthin-starry stain and polymerase chain reaction, were not conducted. Second, although vascular proliferation was identified by H\&E staining, CD31 immunohistochemistry, a confirmatory marker of vascular proliferation, was not performed [15].

In conclusion, dogs with diffuse skin lesions and a hemangioma pattern on histopathology can be diagnosed with cutaneous angiomatosis after excluding other possible diseases, including cutaneous infectious diseases, hemangioma, hemangiosarcoma, and vasculitis. This report presents the first case of canine cutaneous angiomatosis in Korea.

\section{Acknowledgments}

This work was supported by the National Research Foundation of Korea (NRF) grant funded by the Korea government (MSIT) (No. 2021R1A2C1012058).

\section{ORCID}

Yoonhoi Koo, https://orcid.org/0000-0002-3810-4193

Taesik Yun, https://orcid.org/0000-0003-1372-4430

Yeon Chae, https://orcid.org/0000-0002-9816-6900

Dohee Lee, https://orcid.org/0000-0001-7162-9592

Hakhyun Kim, https://orcid.org/0000-0002-8882-2329

Mhan-Pyo Yang, https://orcid.org/0000-0002-8043-0152

Byeong-Teck Kang, https://orcid.org/0000-0002-4471-4342

\section{References}

1. Gross TL, Ihrke P, Walder Ej, Affolter VK. Vascular tumors. In: Gross TL, Ihrke P, Walder Ej, Affolter VK (eds.). Skin Diseases of the Dog and Cat: Clinical and Histopathologic Diagnosis. 2nd ed. pp. 735-758, Blackwell Science Ltd., Oxford, 2005.
2. Kim Y, Reinecke S, Malarkey DE. Cutaneous angiomatosis in a young dog. Vet Pathol 2005;42:378-381.

3. Luppi MM, Malta MC, Ocarino NM, França SA, Serakides R. Cutaneous angiomatosis in a llama (Lama glama). J Comp Pathol 2010;142:223-227.

4. Yager JA, Best SJ, Maggi RG, Varanat M, Znajda N, Breitschwerdt EB. Bacillary angiomatosis in an immunosuppressed dog. Vet Dermatol 2010;21:420-428.

5. Ide K, Uchida N, Iyori K, Mochizuki T, Fukushima R, Iwasaki T, Nishifuji K. Multi-system progressive angiomatosis in a dog resembling blue rubber bleb nevus syndrome in humans. J Small Anim Pract 2013;54:201-204.

6. Laganga P, Marconato L, Cancedda S, Leone VF, Rohrer-Bley C, Rossi F. Radiation therapy for the treatment of canine progressive cutaneous angiomatosis: description of 2 cases. Can Vet J 2018;59:1067-1070.

7. Olivieri L, Nardini G, Pengo G, Abramo F. Cutaneous progressive angiomatosis on the muzzle of a dog, treated by laser photocoagulation therapy. Vet Dermatol 2010;21:517-521.

8. Peavy GM, Walder EJ, Nelson JS, Rosenberg M. Use of laser photocoagulation for treatment of cutaneous angiomatosis in one dog and two cats. J Am Vet Med Assoc 2001;219:10941097.

9. Sluiter KL, Randell SC, Ramirez JR, Farina LL. Bacterial sepsis resulting in severe systemic illness and euthanasia in a dog with cutaneous angiomatosis. Can Vet J 2013;54:397-402.

10. Bulman-Fleming JC, Gibson TW, Kruth SA. Invasive cutaneous angiomatosis and thrombocytopenia in a cat. J Am Vet Med Assoc 2009;234:381-384.

11. Khan S, Pujani M, Jetley S, Neogi S. Angiomatosis: a rare vascular proliferation of head and neck region. J Cutan Aesthet Surg 2015;8:108-110.

12. Hiromatsu Y, Toda S. Mast cells and angiogenesis. Microsc Res Tech 2003;60:64-69.

13. Woldemeskel M, Rajeev S. Mast cells in canine cutaneous hemangioma, hemangiosarcoma and mammary tumors. Vet Res Commun 2010;34:153-160.

14. Hnilica KA, Patterson AP. Neoplastic and Nonneoplastic Tumors. In: Hnilica KA, Patterson AP (eds.). Small Animal Dermatology: A Color Atlas and Therapeutic Guide. 4th ed. pp. 475-477, Elsevier, St. Louis, 2017.

15. Prinz Vavricka BM, Barry C, Victor T, Guitart J. Diffuse dermal angiomatosis associated with calciphylaxis. Am J Dermatopathol 2009;31:653-657. 